\title{
Light- and magnetically actuated FePt microswimmers
}

Vincent Mauricio Kadiri ${ }^{1,2}$ (1), Jan-Philipp Günther ${ }^{1,2}$ (D), Sai Nikhilesh Kottapalli ${ }^{1,2}$ (I) , Rahul Goyal ${ }^{1}$ (D), Florian Peter ${ }^{1,2}\left(\mathbb{D}\right.$, Mariana Alarcón-Correa ${ }^{1} \mathbb{D}$, Kwanghyo Son ${ }^{1} \mathbb{D}$, Hannah-Noa Barad ${ }^{1}$ (D) Michael Börsch ${ }^{3}$ (D), and Peer Fischer ${ }^{1,2, a} \mathbb{B}_{0}$

1 Max Planck Institute for Intelligent Systems, Heisenbergstr. 3, 70569 Stuttgart, Germany

2 Institute of Physical Chemistry, University of Stuttgart, Pfaffenwaldring 55, 70569 Stuttgart, Germany

3 Jena University Hospital, Friedrich-Schiller University Jena, Nonnenplan 4, 07743 Jena, Germany

Received 27 February 2021 / Accepted 29 April 2021 / Published online 2 June 2021

(C) The Author(s) 2021

\begin{abstract}
Externally controlled microswimmers offer prospects for transport in biological research and medical applications. This requires biocompatibility of the swimmers and the possibility to tailor their propulsion mechanisms to the respective low Reynolds number environment. Here, we incorporate low amounts of the biocompatible alloy of iron and platinum (FePt) in its $\mathrm{L} 1_{0}$ phase in microstructures by a versatile one-step physical vapor deposition process. We show that the hard magnetic properties of $\mathrm{L} 1_{0}$ FePt are beneficial for the propulsion of helical micropropellers with rotating magnetic fields. Finally, we find that the FePt coatings are catalytically active and also make for Janus microswimmers that can be light-actuated and magnetically guided.
\end{abstract}

\section{Introduction}

Biomedical applications of micro- and nanoparticulate carriers based on liposomes and viruses or virus-like particles mostly rely on passive diffusion to reach their target site $[1,2]$. Such systemic applications require larger doses and are generally wasteful as most carriers do not reach their intended target [2]. It is therefore of interest to ask whether biocompatible actively propelled micro- or nanocarriers can enable targeted delivery.

If synthetic analogues are to propel like swimming microorganisms, they have to do so under the constraints of low Reynolds number hydrodynamics $(\operatorname{Re}<$ 1 ), where the viscous forces dominate over inertial forces [3]. Active swimming in Newtonian fluids, like water, thus requires propulsion mechanisms based on non-reciprocal motion to overcome the scallop theorem [3]. An exception is only provided in non-Newtonian viscoelastic fluids, where even a reciprocal motion can lead to propulsion [4]. While it is challenging to develop force-free microswimmers that propel by metachronal body shape changes [5], it is also possible to mimic the flagellar motion of bacteria by exerting a torque on helical micro- and nanopropellers with the help of an external rotating magnetic field (see Fig. 1a) $[6,7]$. These helical structures have also been successfully propelled through biological fluids by decreasing their size in order to pass through the macromolecular mesh of a hyaluronan gel [8], by coating them with enzymes such that they can liquefy mucines [9] or by coating them with non-adhesive perfluorocarbons so that they can be moved through the vitreous body of the eye [10]. In all these cases, the propellers require a hard ferromagnetic section that can be used to propel the structures by rotation-translation coupling.

The alloying of platinum and iron can yield such a hard magnet, which in addition is non-toxic, unlike many other magnetic materials $[11,12]$. FePt must be in the $\mathrm{L} 1_{0}$ phase to exhibit these favorable magnetic properties. As such, $\mathrm{L}_{0} \mathrm{FePt}$ has garnered significant attention as a non-cytotoxic, rare earth-free, hard magnetic material with some of the highest energy products observed to date $[13,14]$. As we demonstrated recently, $\mathrm{L} 1_{0} \mathrm{FePt}$ microhelices are not cytotoxic and are thus suitable for biomedical applications $[12,14]$. The possibility to form nanostructures of $\mathrm{L} 1_{0} \mathrm{FePt}$ via the co-deposition of iron and platinum in a physical vapor deposition chamber at glancing angles opens further avenues for the application in microdevices, two of which are explored here. First, we show that reducing the FePt coating thickness can improve the magnetic properties. In addition, we show that the FePt coating is also catalytically active. This opens the possibility to realize light-actuated iron-platinum microswimmers that are propelled by self-phoresis (see Fig. 1b) [15].

\footnotetext{
a e-mail: fischer@is.mpg.de (corresponding author)
} 
a

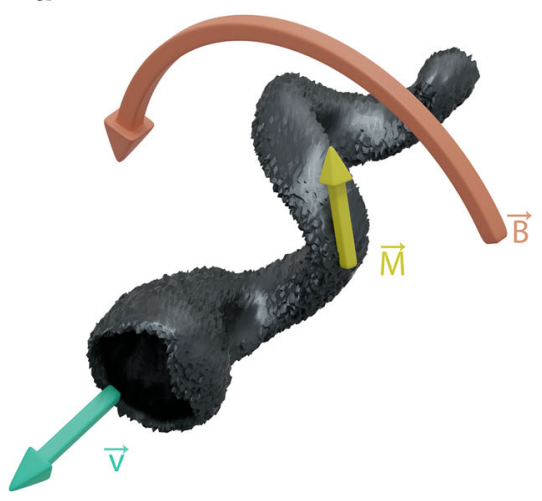

Fig. $1 \mathrm{~L} 1_{0} \mathrm{FePt}$ is a versatile hard magnetic material that can be nanostructured to yield a helices propelled by a rotating magnetic field ([12] and this work) and $\mathbf{b}$ light-actuated Janus particles propelled by self-phoresis and directed by a

\section{Methods}

\subsection{Fabrication of $\mathrm{L}_{0}$ FePt Janus particles and helices}

FePt-coated helical microswimmers were fabricated on a monolayer of $1 \mu \mathrm{m}$ polystyrene beads. $1 \mathrm{~mL}$ polystyrene beads (2.5 wt\%, Kisker Biotech) suspended in water were centrifuged at $14000 \mathrm{rcf}$ for 5 minutes, and the supernatant was removed and then re-dispersed in deionized water. This cleaning step was repeated and an equal volume of ethanol was added. The particles were assembled at the air/water interface of a $0.1 \mathrm{mM}$ sodium dodecyl sulfate (SDS) solution. The dispersion was slowly pipetted onto a silicon slide partially submerged at a $45^{\circ}$ angle in the liquid phase, to create a particle monolayer at the air-liquid interface. The resulting monolayer was transferred onto a fresh silicon wafer via an automated Langmuir-Blodgett system [16]. After drying, the substrate was exposed to an oxygen plasma (200 W, 0.7 mbar) for 15 minutes to reduce the size of the beads to $500-600 \mathrm{~nm}$. Onto these seed particles, $1.5 \mu \mathrm{m} \mathrm{SiO}_{2}$ helices were grown via glancing angle deposition (GLAD) at a deposition angle of $83^{\circ}[16,17]$. A $5 \mathrm{~nm}$ adhesion layer of Ti was grown at $18^{\circ}$ before $\mathrm{Fe}$ and $\mathrm{Pt}$ were co-deposited at a 50:50 atomic ratio at a deposition angle of $18^{\circ}$ while rotating the substrate. The FePt layer was grown with thicknesses between $50 \mathrm{~nm}$ and $200 \mathrm{~nm}$. Finally, the wafer was annealed in a vacuum ampoule at $680{ }^{\circ} \mathrm{C}$ for $1 \mathrm{~h}$.

Janus particles were fabricated in a similar way to those fabricated with $\mathrm{Pt}-\mathrm{SiO}_{2}[18,19]$. Briefly, $1 \mu \mathrm{m}$ $\mathrm{SiO}_{2}$ beads were transferred onto a silicon wafer via Langmuir-Blodgett deposition on which a $5 \mathrm{~nm} \mathrm{Ti}$ adhesion layer was first grown to minimize the lattice

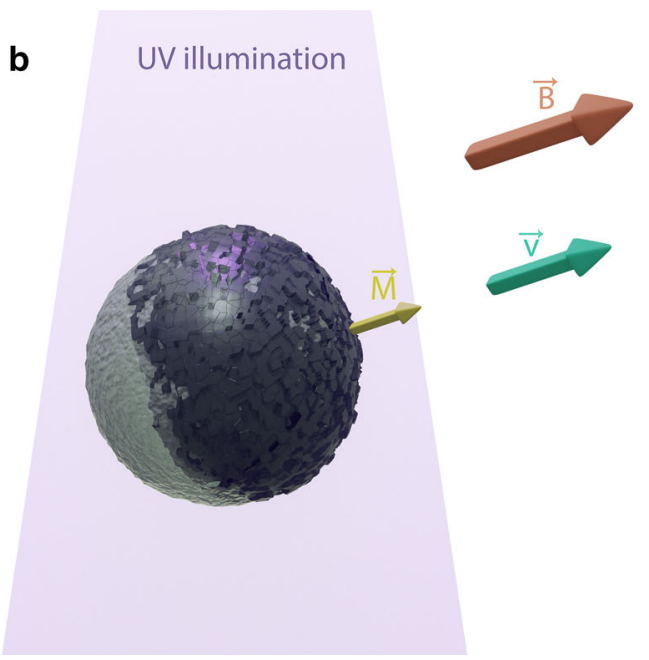

static magnetic field (this work). The propulsion velocity $\vec{v}$ is pictured in green, remanent magnetization $\vec{M}$ is shown in yellow, applied (rotating) magnetic field $\vec{B}$ in orange and UV illumination in purple

mismatch between $\mathrm{SiO}_{2}$ and FePt. A thin film of 50:50 co-deposited $\mathrm{Fe}$ and $\mathrm{Pt}$ was then evaporated and the wafer was subsequently annealed in a vacuum ampoule at $680{ }^{\circ} \mathrm{C}$ for $1 \mathrm{~h}$ to yield $\mathrm{L} 1_{0}$ FePt-coated Janus particles.

Scanning electron micrographs were acquired on a Zeiss Gemini scanning electron microscope (SEM).

Energy-dispersive X-ray photoelectron spectroscopy (EDX) scans were performed on a Zeiss SESAM transmission electron microscope at $200 \mathrm{kV}$ with an EDX Noran system and Pathfinder 1.1 software.

\subsection{SQUID magnetometry}

The magnetic properties of wafer pieces with $\mathrm{FePt}$ helices (annealed) and Janus particles (as-deposited, annealed, and aged) were characterized via superconducting quantum interference device (SQUID, MPMS7, quantum design) magnetometry. The field-dependent magnetization measurements were conducted at room temperature and the field was varied from $7 \mathrm{~T}$ to $-7 \mathrm{~T}$. For the $M-\mathrm{H}$ hysteresis loop measurements, the external magnetic field $\mathrm{H}$ has been applied in the out-of-plane and the in-plane directions where in-plane denotes a measurement perpendicular to the wafer surface's normal while out-of-plane is defined as parallel to it. The contribution of the substrate and the $\mathrm{SiO}_{2}$ was subtracted via linear fitting at high field ranges.

\subsection{Oxygen evolution}

Wafer pieces $\left(\sim 2 \mathrm{~cm}^{2}\right)$ of annealed and as-deposited $\mathrm{FePt}$ Janus particles were submerged in a $10 \%(\mathrm{w} / \mathrm{v})$ $\mathrm{H}_{2} \mathrm{O}_{2}$ solution inside a round-bottomed flask. The gas evolution was quantified with and without UV light with a measuring cylinder over time via displacement 
Table 1 Comparison of magnetic properties of $\mathrm{L} 1_{0}$ FePt helices for different fabrication schemes, including the optimized method of this paper

\begin{tabular}{llllll}
\hline $\begin{array}{l}\text { Nominal } \\
\text { deposition } \\
\text { thickness }\end{array}$ & $\begin{array}{l}\text { Deposition } \\
\text { angle } \alpha\end{array}$ & $\begin{array}{l}\text { Remanence } \\
M_{\mathrm{R}} \frac{10^{-6} \mathrm{emu}}{\mathrm{mm}^{2}}\end{array}$ & $\begin{array}{l}\text { Saturation } M_{\mathrm{S}} \\
\frac{10^{-6} \mathrm{emu}}{\mathrm{mm}^{2}}\end{array}$ & $\begin{array}{l}\text { Remanence } \\
M_{\mathrm{R}} \frac{10^{-12} \mathrm{emu}}{\mathrm{helix}}\end{array}$ & $\begin{array}{l}\text { Saturation } \\
\frac{10^{-12} \mathrm{emu}}{\mathrm{helix}}\end{array}$ \\
\hline $500 \mathrm{~nm}[12]$ & $83^{\circ}$ & 11.2 & 12.2 & 2.4 & 2.6 \\
$100 \mathrm{~nm}$ & $45^{\circ}$ & $22.9 \pm 0.2$ & $34.3 \pm 0.9$ & 19.8 & 29.7 \\
$200 \mathrm{~nm}$ & $18^{\circ}$ & $65.9 \pm 0.2$ & $101.2 \pm 0.4$ & 57.1 & 87.6 \\
$100 \mathrm{~nm}$ & $18^{\circ}$ & $37.0 \pm 5.0$ & $52.0 \pm 1.0$ & 32 & 45 \\
$50 \mathrm{~nm}$ & $18^{\circ}$ & $18.9 \pm 0.5$ & $27 \pm 7$ & 16.4 & 23.4 \\
\hline
\end{tabular}

of the water by the $\mathrm{O}_{2}$ gas. UV illumination was controlled via a UV LED (M365LP1-365 nm, $2000 \mathrm{~mW}$, $1700 \mathrm{~mA}$, ThorLabs).

\subsection{Particle tracking}

Janus particles were removed from the wafer using sonication and then dispersed in solutions with $\mathrm{H}_{2} \mathrm{O}_{2}$ concentrations of 0,2 and $5 \%(\mathrm{w} / \mathrm{v})$. The particles were imaged with a Zeiss Axiovert microscope using a $40 \mathrm{X}$ objective with and without UV light $\left(320 \mathrm{~mW} \mathrm{~cm}^{-2}\right)$. Images were acquired at $15 \mathrm{fps}$ for 60 seconds and particle diffusion was evaluated via a custom python script based on TrackPy [20,21], and MSD data fitted to obtain the effective diffusion coefficients $D[22]$.

\subsection{Magnetic actuation}

The magnetic helices were magnetized in-plane with a $2 \mathrm{~T}$ electromagnet while they were still attached to the wafer. Then the magnetized helices were sonicated to yield final concentrations of $10^{5}$ particles $/ \mathrm{mL}$ and actuated as described in previous publications $[6,10]$. A frequency sweep using rotating magnetic fields with strengths of 2 and $4 \mathrm{mT}$ was performed and imaged on a Zeiss Axiovert microscope using a $63 \mathrm{X}$ objective. The magnetic field was applied via a custom-built 3axis Helmholtz coil [16].

Similarly, Janus particles were magnetized out-ofplane and magnetically guided using the same setup with a static, homogeneous magnetic field of $3.5 \mathrm{mT}$.

\subsection{ARPE-19 cell culture}

ARPE-19 cells were cultured in Dulbecco's modified Eagle medium (DMEM $1 \mathrm{~g} / \mathrm{L}$ Glucose, Gibco) with $10 \%(\mathrm{w} / \mathrm{v})$ fetal bovine serum (FBS, Gibco) and 1\% $(\mathrm{w} / \mathrm{v})$ Penicillin-Streptomycin. Cells were incubated in an atmosphere of $5 \% \mathrm{CO}_{2}$ at $37^{\circ} \mathrm{C}$ and passaged every 7 days at a ratio of 1:7.

\subsection{Cell viability}

Cell viability in the presence of FePt microswimmers was analyzed via a modified standard protocol of
"LIVE/DEAD"TM Fixable Red Dead Cell Stain Kit, for 488 nm excitation" (Invitrogen). ARPE-19 cells were incubated with a 100:1 ratio of particles to cells. After incubation for 24 or $72 \mathrm{~h}$, LIVE/DEAD ${ }^{\mathrm{TM}}$ Fixable Red Dead Cell Stain was added in a ratio of $1 \mu \mathrm{L}$ for every $\sim 10^{6}$ cells and incubated for $30 \mathrm{~min}$. The medium was then aspirated and the cells washed with PBS, fixed with a $3.7 \%(\mathrm{w} / \mathrm{v})$ paraformaldehyde solution and washed with PBS three more times. The cells were permeabilized with $0.1 \%(\mathrm{w} / \mathrm{v})$ Triton-X solution. Immunostaining was then performed with DAPI $\left(4^{\prime}, 6\right.$ diamidino-2-phenylindole, Invitrogen). The cells were subsequently imaged on a Zeiss LSM 900 Airyscan microscope with a $20 \mathrm{X}$ objective, and fluorescence data were extracted and evaluated via a custom-written CellProfiler pipeline [23].

\section{$2.8 \times P S$}

X-ray photoelectron spectroscopy (XPS) measurements were taken on the sample using a Theta Probe AngleResolved X-ray Photoelectron Spectrometer System (Thermo Fisher Scientific Inc.). For the excitation, a non-monochromatic Al K $\alpha$ source was used, which was kept at $100 \mathrm{~W}(\mathrm{~h} v=1486.68 \mathrm{eV})$. The survey scan was acquired with an energy pass of $200 \mathrm{eV}$, followed by high-resolution spectra acquisition of the various elements using an energy pass of $50 \mathrm{eV}$. The quantitative analysis was executed using Avantage software; at first, a nonlinear Shirley background was removed from the high-resolution core-level spectra of the elements; then, the peak analysis and fitting were done using the Powell method. The charge referencing was corrected by adjusting the $\mathrm{C}-\mathrm{C}$ (or $\mathrm{C}-\mathrm{H}$ ) component of the $\mathrm{C} 1 \mathrm{~s}$ peak to $284.8 \mathrm{eV}$.

\section{Results and discussion}

\subsection{Magnetically driven FePt microswimmers}

We first show that the magnetic properties of helical microswimmers have been optimized by an improved fabrication method. In contrast to the direct deposition of Fe and Pt onto silica helices [12], the optimized FePt helices of this work require one-tenth of the material during deposition while enabling an eightfold increase 
Fig. 2 a Optimized fabrication scheme to generate, $\mathbf{b}$ helices (scanning electron micrograph) with higher magnetizations. The scale bar is $500 \mathrm{~nm}$
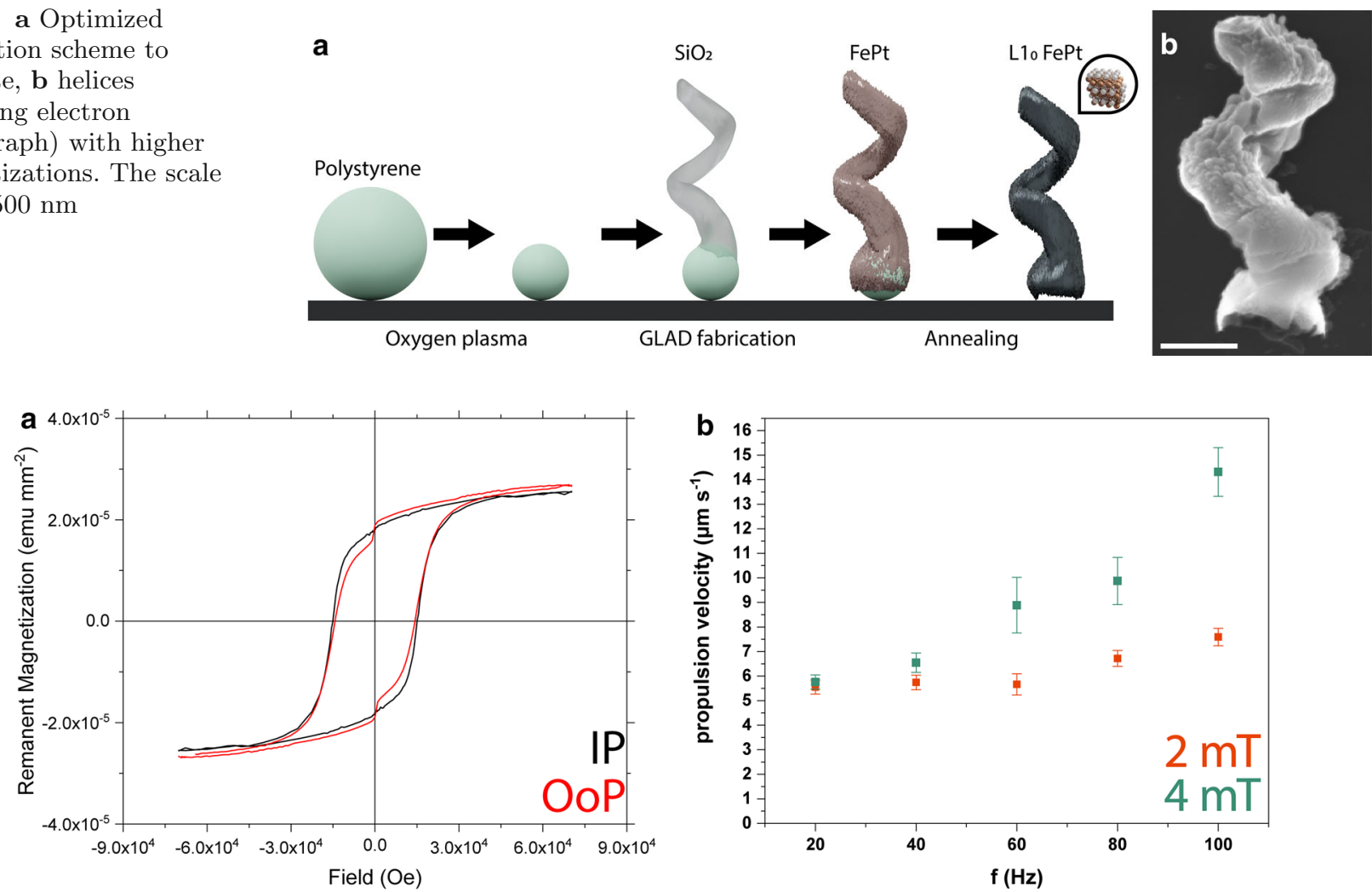

C

Fig. 3 a Magnetic hysteresis curve for optimized helical microswimmers $\left(50 \mathrm{~nm}, 18^{\circ}\right)$. b Propulsion velocities for frequency sweeps with a magnetic field of 2 (orange) and $4 \mathrm{mT}$ (green), respectively. c Optimized helical microswimmers spelling out "FePt." Scale bar is $50 \mu \mathrm{m}$

in remanent magnetization per helix (see Table 1). Two effects may explain this significant increase in magnetic performance: firstly, a new deposition scheme, which is schematically shown in Fig. 2a, and secondly, the reduction in the thickness of the FePt coating. Previously, FePt helices were fabricated by depositing $\mathrm{SiO}_{2}$ helices onto a $500 \mathrm{~nm} \mathrm{SiO}_{2}$ bead seed layer on which FePt, at a nominal thickness of $500 \mathrm{~nm}$ and an angle of $83^{\circ}$, was then grown. In this work, we use 500-600 nm polystyrene beads (after oxygen plasma etching), which are spaced $\sim 1 \mu \mathrm{m}$ apart, as seeds (see Experimental section and Fig. 2a). After growing the $1.5 \mu \mathrm{m} \mathrm{SiO}_{2}$ helix, between 50 and $200 \mathrm{~nm} \mathrm{FePt}$ is co-deposited and annealed (see Fig. 3). Crucially, the FePt is not deposited under oblique incidence, but at a reduced angle. Following Hawkeye et al. [17], this shadowing constraint follows from the deposition geometry:

$$
h \tan \alpha \leq d-w
$$

where $h$ is the height of the growing particle, $\alpha$ the deposition angle, $d$ the center-to-center distance of seed particles and $w$ the width of the particle. In the present geometry, $h=1.5 \mu \mathrm{m}, w=0.5 \mu \mathrm{m}, d=1 \mu \mathrm{m}$, which yields a deposition angle of $\alpha=18^{\circ}$. This reduced angle allows for the incident vapor to penetrate in between 
Fig. 4 a Fabrication of photocatalytic $\mathrm{L} 1_{0} \mathrm{FePt}$ Janus particles and scanning electron micrographs, $\mathbf{b}$ before and c after annealing at $680{ }^{\circ} \mathrm{C}$ and sonication. Scale bars are $1 \mu \mathrm{m}$
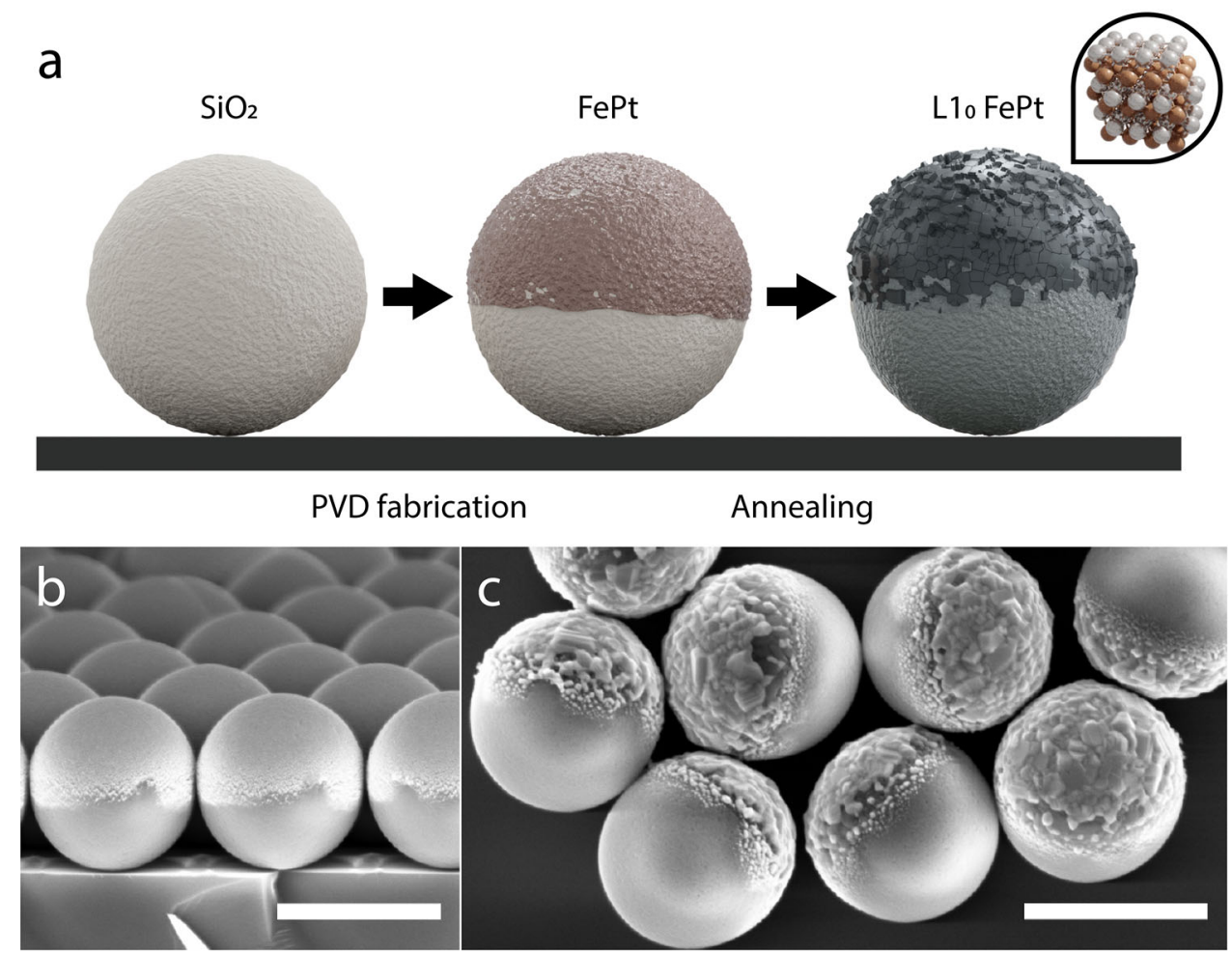

the helices while avoiding inter-seed deposition onto the substrate.

As a result of coating the helices at $18^{\circ}$ instead of $83^{\circ}$, the entire helices are covered with a thin layer of FePt. While this means that it is more difficult to estimate the magnetization per volume $\left(\mathrm{emu} \mathrm{cm}^{-3}\right)$, the magnetization per surface area $\left(\mathrm{emu} \mathrm{mm}^{-2}\right)$ or per helix can still be determined accurately. It should be noted that because of the new deposition scheme, the amount of helices covering the wafer's surface per $\mathrm{mm}^{2}$ is reduced by a factor of $4\left(\sim 2.5 \times 10^{9}\right.$ particles per 2 inch wafer) compared to samples that are fabricated on close-packed seed layers. Nevertheless, the samples grown at $18^{\circ}$ with a nominal FePt thickness of $50 \mathrm{~nm}$ still exhibit an almost twofold increase in

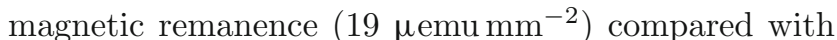
the helices that have previously been grown at $83^{\circ}$ with a nominal thickness $500 \mathrm{~nm}$, which only showed $11.16 \mu \mathrm{emu} \mathrm{mm}^{-2}$. In other words, changing the deposition scheme optimizes material use by a factor of 20 and a factor of 80 when evaluating on a per helix basis. Beside this, the magnetic coating's curved geometry may also have a favorable effect on the magnetization [24]. Table 1 shows that the deposition of material up to a thickness of $200 \mathrm{~nm}$ at $18^{\circ}$ leads to an increase in remanence. Depositing more than $200 \mathrm{~nm}$ would lead to the fusion of helices and was thus not investigated. We find that depositing at higher angles leads to more material loss and lower magnetization, while the increase in remanence levels off. The latter may be explained by the critical domain size for FePt. Above the critical domain size, $\mathrm{L} 1_{0} \mathrm{FePt}$ nanomagnets no longer possess a single domain structure. Kikuchi et al. observed a critical domain size of $50 \mathrm{~nm}$ for $10 \mathrm{~nm}$ FePt layers [25]. One would thus expect that while the saturation magnetization increases beyond thicknesses of $50 \mathrm{~nm}$, the remanence will level off, as is observed in Table 1 (and [12]). The helices in this work (see Fig. $2 \mathrm{~b}$ ) are coated with a thin $\mathrm{L} 1_{0} \mathrm{FePt}$ film that covers the entire helix, as compared to helices with a $350 \mathrm{~nm}$ magnetic section that were used previously and that showed a much smaller remanence (see Table 1 and [12]).

As the hysteresis curve in Fig. 3a illustrates, the FePt helices retain their hard magnetic behavior despite the reduction in the thickness of the FePt coating compared to previous work.

Depositing a thinner layer of FePt also did not lead to a significant decrease in propulsion speeds, as shown in Fig. 3b for rotating external fields of 2 and $4 \mathrm{mT}$. In contrast to previous FePt helical microswimmers [12], we did not detect a step-out frequency below $100 \mathrm{~Hz}$ for either field strength. We associate this observation with the higher magnetic moment [26]. Similarly, the maneuverability was unaffected (Fig. 3c and Supplementary Video 1). Thus, optimized $\mathrm{L} 1_{0} \mathrm{FePt}$ helices conserve material without sacrificing the magnetic performance of these actuated microstructures.

\subsection{Catalytic microswimmers based on FePt}

Apart from the magnetic properties, FePt nanoparticles have previously shown catalytic activity in heterogeneous Fenton-like reactions [27]. Moreover, both iron oxides and platinum are commonly used (green) catalysts $[18,27-29]$, and thus, it is interesting to examine 

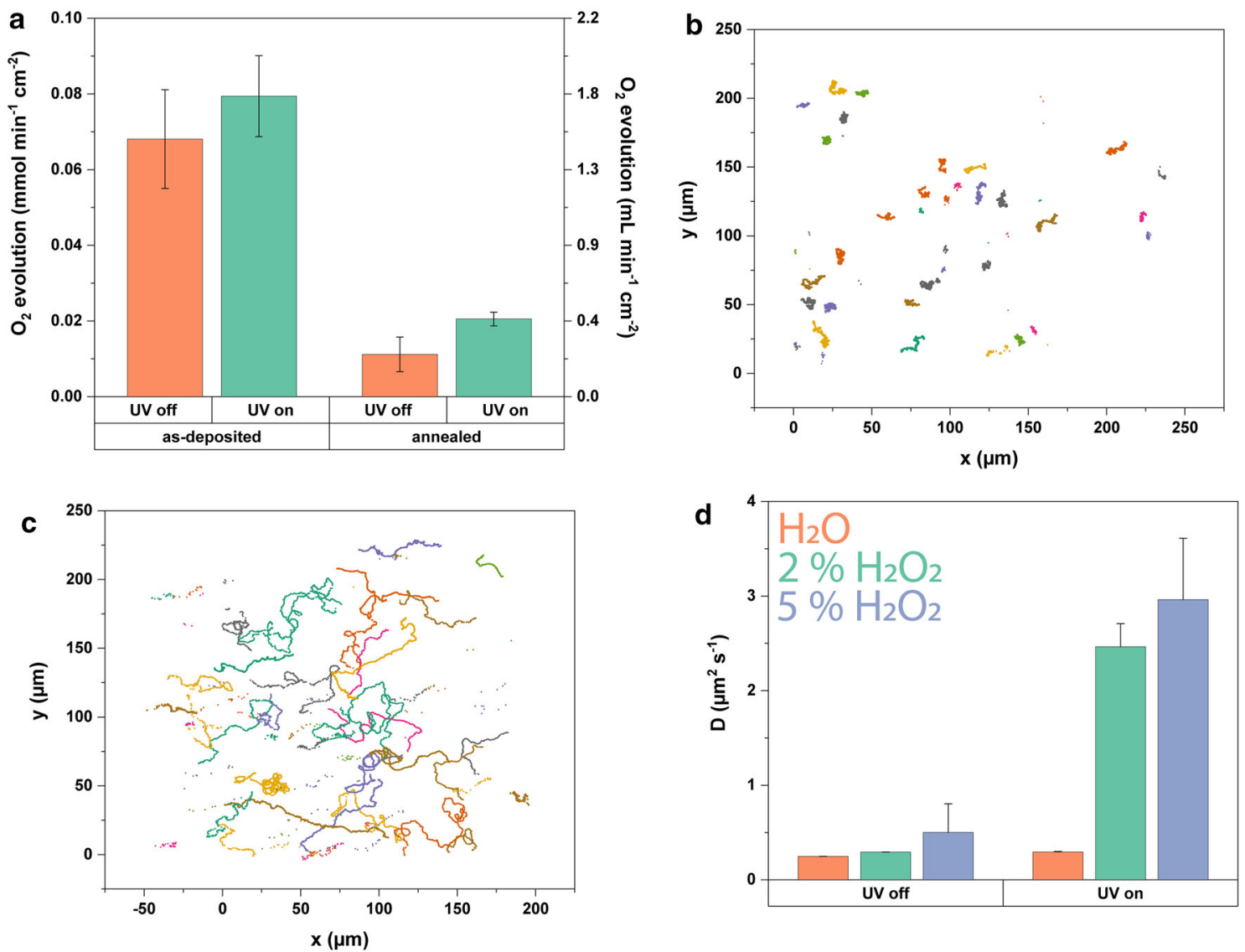

Fig. 5 a FePt-coated wafers catalyze the decomposition of hydrogen peroxide. In the case of annealed $\mathrm{L} 1_{0} \mathrm{FePt}$, UV irradiation leads to a twofold increase in activity. Particle tracking data confirms this effect for FePt-Ti- coated Janus particles in $2 \%$ (w/v) $\mathrm{H}_{2} \mathrm{O}_{2}$ solutions, which diffuse

whether the FePt-coated structures also display catalytic activity. This would permit the realization of chemically propelled self-phoretic microswimmers that could also be magnetically guided by virtue of the $\mathrm{L} 1_{0}$ FePt. The versatility of the FePt nanostructuring allows for the facile fabrication of Janus particles based on $\mathrm{SiO}_{2}$ with a $5 \mathrm{~nm}$ Ti adhesion layer as shown in Fig. $4 \mathrm{a}-\mathrm{c}$. The as-fabricated particles were immersed in a solution containing $10 \%(\mathrm{w} / \mathrm{v}) \mathrm{H}_{2} \mathrm{O}_{2}$ and the oxygen gas was quantified with the aid of a measuring cylinder [18].

The as-deposited $50 \mathrm{~nm}$ film of FePt was initially found to catalyze $\mathrm{H}_{2} \mathrm{O}_{2}$ decomposition at a rate of $0.07 \pm 0.01 \mathrm{mmol}(=1.5 \mathrm{~mL})$ per min and $\mathrm{cm}^{2}$ of wafer (see Fig. 5a). For reference, Choudhury et al. [18] previously reported a $\mathrm{Pt}$ film of $20 \mathrm{~nm}$ nominal thickness catalyzing the same reaction at a rate of $1 \mathrm{mmol}(=22.4$ $\mathrm{mL}$ ) $\mathrm{min}^{-1} \mathrm{~cm}^{-2}$. Thus, the co-deposition of $\mathrm{Pt}$ with $\mathrm{Fe}$ certainly leads to a decrease in reactivity. Annealing the wafer to obtain $\mathrm{L}_{0} \mathrm{FePt}$ led to a further drop in activity. Nevertheless, a small activity of $0.01 \mathrm{mmol}(=0.2$ $\mathrm{mL}) \mathrm{min}^{-1} \mathrm{~cm}^{-2}$ of $\mathrm{O}_{2}$ was still measured.

slower $\mathbf{b}$ without UV compared to $\mathbf{c}$ those with UV irradiation. d Effective diffusion coefficients obtained from particle tracking show a significant diffusion enhancement upon UV illumination in $\mathrm{H}_{2} \mathrm{O}_{2}$

At first glance, it would thus seem that the excellent magnetic and the catalytic properties of FePt are mutually exclusive. Surprisingly, we did find, however, that irradiation with UV light led to a photo-switchable statistically significant twofold increase in oxygen evolution (Fig. 5a). Over the course of multiple experiments, a permanent increase of activity after UV illumination was also observed. The UV light does not lead to a significant increase of oxygen evolution on the asdeposited FePt. Thus, the annealing step, which is also crucial for generating the as-prepared $\mathrm{L} 1_{0}$ phase $\mathrm{FePt}$ coating, seems important for this photocatalytic effect, too.

After sonicating the particles off the wafer, they were dispersed in 2 and $5 \%(\mathrm{w} / \mathrm{v}) \mathrm{H}_{2} \mathrm{O}_{2}$ solutions to examine if $\mathrm{L}_{0} \mathrm{FePt}-\mathrm{Ti}-\mathrm{SiO}_{2}$-based Janus particles self-propel. Supplementary Videos 2 and 3, respectively, depict the Janus particles without and with UV illumination at 2 $\% \mathrm{H} 2 \mathrm{O} 2$. Tracking data of these videos is also displayed in Fig. 5b, c. Supplementary Videos 4 and 5 are at 5 $\% \mathrm{H} 2 \mathrm{O} 2$. Dispersing the particles in hydrogen peroxide solutions results in enhanced diffusion upon UV irradiation. As expected, the $\mathrm{L} 1_{0}$ FePt Janus particles dis- 

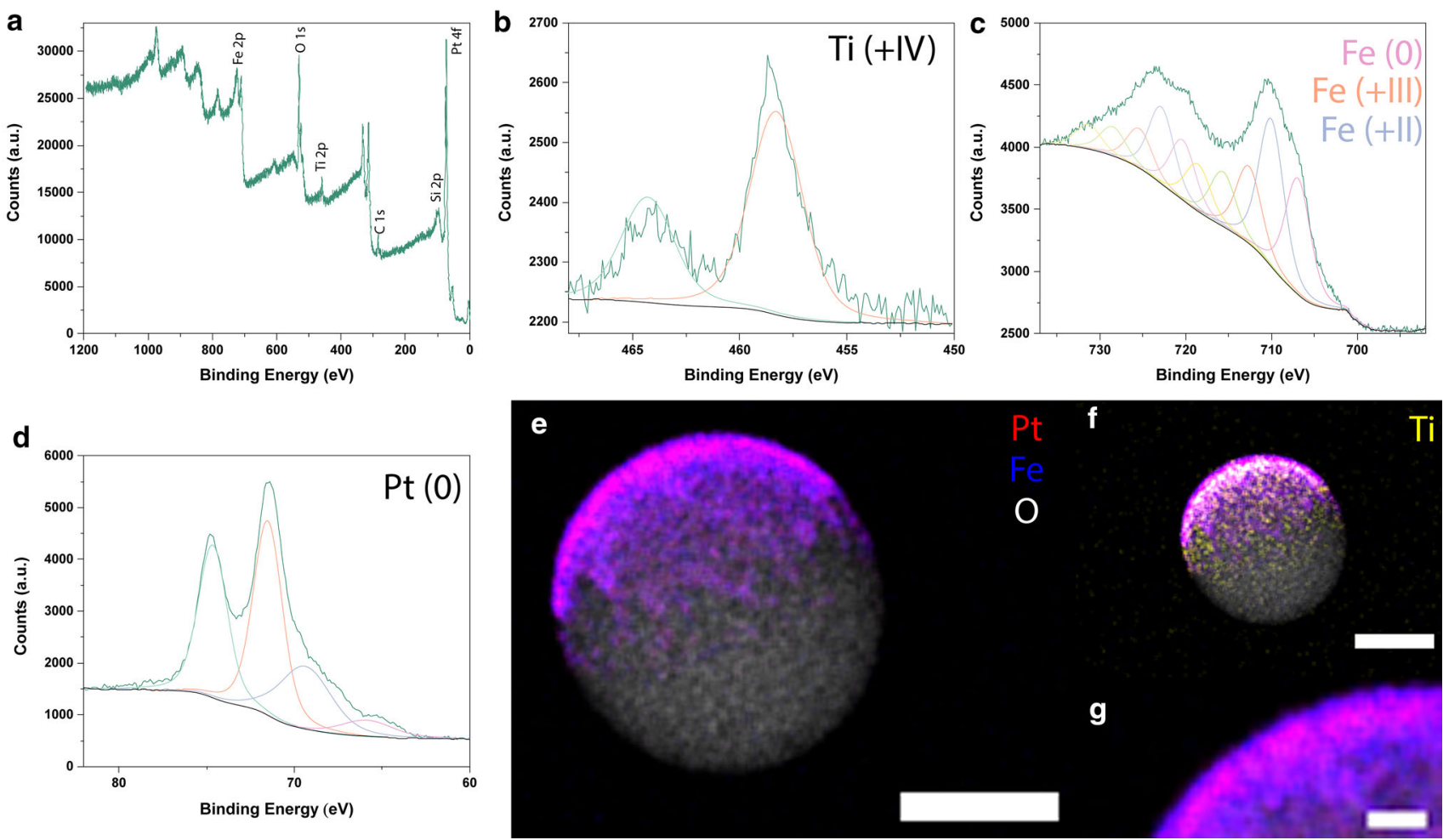

Fig. 6 a Surface characterization via X-ray photoelectron spectroscopy (XPS) yielded information on oxidation states of elements present in L10 FePt Janus particles. In b, Titanium was exclusively present as $\mathrm{Ti}(+\mathrm{IV})$, while in $\mathbf{c}$ the iron species were found to be present in the oxidation states $(0)$, $(+\mathrm{II})$ and (+III), suggesting the presence of both metallic iron and iron oxides. d Platinum was not oxidized. e
Energy-dispersive X-ray spectroscopy (EDX) imaging in a transmission electron microscope (TEM) showing the location of Fe (blue), Pt (red), O (white) and $\mathbf{f} \mathrm{Ti}$ (yellow) on the Janus particles. Scale bars are $500 \mathrm{~nm}$. g Distribution of $\mathrm{Fe}$ around the outer regions of the FePt layer. Scale bar is $50 \mathrm{~nm}$ persed in water showed no significant increase in their diffusion coefficient upon UV irradiation (Fig. 5d).

X-ray photoelectron spectroscopy (XPS) was conducted to characterize the $\mathrm{L}_{0}$ FePt-based Janus particles as well as shed light on a possible mechanism underlying the photocatalytic properties. Iron, oxygen, titanium, carbon, silicon and platinum peaks were all readily identified (Fig. 6a), and the overall Fe-to-Pt surface ratio was determined to be 52:48. Titanium, which was added as a $5 \mathrm{~nm}$ adhesion layer between the silicon dioxide particles and the FePt layer, was found at an atomic fraction of $9.7 \%$ when compared to FePt (Fig. $6 \mathrm{~b})$. Titanium was exclusively found with an oxidation state of $(+\mathrm{IV})$ suggesting oxidation via ambient oxygen either during annealing or from the underlying silicon dioxide (probably forming $\mathrm{TiO}_{2}$ ). While platinum was solely present in the oxidation state $(0)$, iron peaks for $\mathrm{Fe}(0), \mathrm{Fe}(+\mathrm{III})$ and $\mathrm{Fe}(+\mathrm{II})$ were identified and fitted (Fig. 6c, d), thereby confirming that the deposited iron must have similarly reacted with oxygen during annealing.

FePt nanoparticles form a thin $(\sim 2 \mathrm{~nm}) \mathrm{FeO}_{\mathrm{x}}$ passivation layer upon annealing, as was recently reported [30]. The high-resolution XPS spectrum of the Fe $2 \mathrm{p}$ levels indicates the presence of $\mathrm{Fe}(+\mathrm{III})$ typically seen in a $\mathrm{Fe}_{2} \mathrm{O}_{3}$ crystal structure [31]. Palacci et al. have shown that $\mathrm{Fe}_{2} \mathrm{O}_{3}$ is photocatalytically active and enables active motion of hematite-based Janus microswimmers, and hence, iron oxide may also underlie the FePt-based particles' photocatalytic activity $[28,32]$.

However, our fabrication scheme (Fig. 4a) also included a $5 \mathrm{~nm}$ Ti adhesion layer, which turned to $\mathrm{TiO}_{2}$ after annealing at $680{ }^{\circ} \mathrm{C}$, i.e., above the annealing temperature for the anatase $\mathrm{TiO}_{2}$ crystal structure [33]. Therefore, one cannot completely rule out that $\mathrm{TiO}_{2}$ contributes to the photocatalytic properties, since anatase- $\mathrm{TiO}_{2}$ is a well-known semiconductor and photocatalyst, with a band gap of $3.2 \mathrm{eV}$. The particles were analyzed via energy-dispersive X-ray spectroscopy (EDX) to better understand the distribution of the $\mathrm{Pt}$ and Fe (Fig. 6e), and Ti species (Fig. 6f) on the particles. From the EDX (and XPS) results, we assume that $\mathrm{Ti}$ is localized more toward the sides of the particles as well as the interface between FePt and silicon dioxide, while both Fe and Pt can be found on the outer surface, with a higher concentration of iron than platinum on the outer layer (Fig. 6g). Combined with the knowledge of the presence of the oxidized $\mathrm{Fe}(+\mathrm{II},+\mathrm{III})$ species at the surface, this could also explain the particle's photocatalytic activity as previously described by Richard 

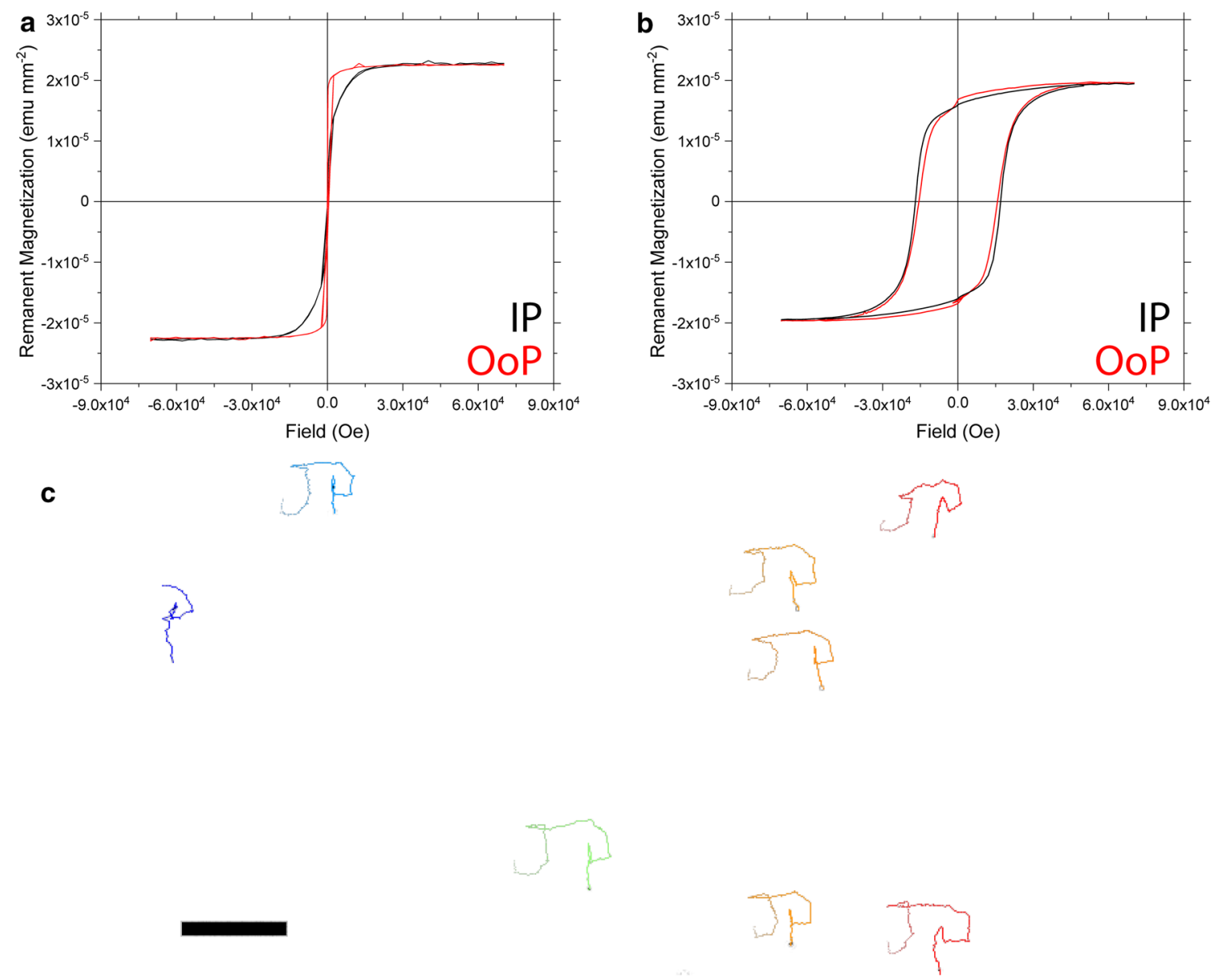

Fig. 7 Magnetization hysteresis curve for FePt-Ti-based Janus particles a before and b after annealing. c Magnetically guided Janus particles spelling out "JP"

et al. [28] and Palacci et al. [32]. However, the exact mechanism still needs to be determined.

As SQUID characterization reveals, the as-deposited Janus particles exhibit a soft magnetic behavior and turn into hard magnets post-annealing (Fig. 7a, b). They can be magnetized out-of-plane, leaving them with a remanent magnetization on the order of 185 emu $\mathrm{cm}^{-3}$. In combination with the observed photoswitchable catalytic behavior, this yields light-activated magnetically steerable microswimmers. When the light is turned on and a static magnetic field is applied, the Janus swimmers will propel along the magnetic field lines (Supplementary Video 6, Fig. 7c). Similar properties were shown for sequentially deposited $\mathrm{Co}$ and $\mathrm{TiO}_{2}[34,35]$. The ability to use $\mathrm{L}_{0} \mathrm{FePt}$, as discussed above, leads to more favorable magnetic properties, but it remains to be established to which extent the Ti and FePt layers, respectively, contribute to the microswimmers' activity.

We found that over the course of 15 UV off-on cycles, the photocatalytic activity was not impaired (Fig. 8a). The particles repeatedly stopped exhibiting enhanced diffusion upon switching the UV illumination off suggesting the particles do in fact exhibit switchable photocatalytic behavior.
Additional XPS studies revealed that after multiple decomposition cycles in $10 \%(\mathrm{w} / \mathrm{v}) \mathrm{H}_{2} \mathrm{O}_{2}$, the $\mathrm{Fe}(0)$ shoulder observed in Fig. 6c disappears while $\mathrm{Fe}(+\mathrm{III})$ species become more prevalent, while platinum becomes partially hydroxylated (Fig. 8b, c). Crucially, the overall magnetization of the particles remains unaffected (Fig. 8d, e). These results suggest that the involvement of $\mathrm{Fe}(0)$ at the particle surface is initially not catalytic since iron undergoes oxidation. Whether the $\mathrm{Fe}(+\mathrm{II}$, + III) at the surface is involved in the catalysis after oxidation remains to be determined. Secondly, since oxidation does not negatively affect the overall hard magnetic properties, the oxidation step seems to be self-limiting, i.e., an iron oxide passivation layer protects the remaining $\mathrm{L}_{0} \mathrm{FePt}$ from being oxidized. Thirdly, since the change of oxidation states of both iron and platinum on the surface (aging) does not seem to interfere with the swimmer' switchable photocatalytic activity (Fig. $8 \mathrm{a})$, it appears likely that the latter is due to the titanium adhesion layer.

To ascertain L1 $1_{0}$ FePt's contribution toward the decomposition of hydrogen peroxide, we thus fabricated identical particles to those depicted in Figs. $4 \mathrm{a}-\mathrm{c}$ and 6e-g without Ti. Oxygen evolution in $10 \%(\mathrm{w} / \mathrm{w}) \mathrm{H}_{2} \mathrm{O}_{2}$ was low at first and comparable to non-aged annealed particles with titanium. Over the course of 45 minutes, 

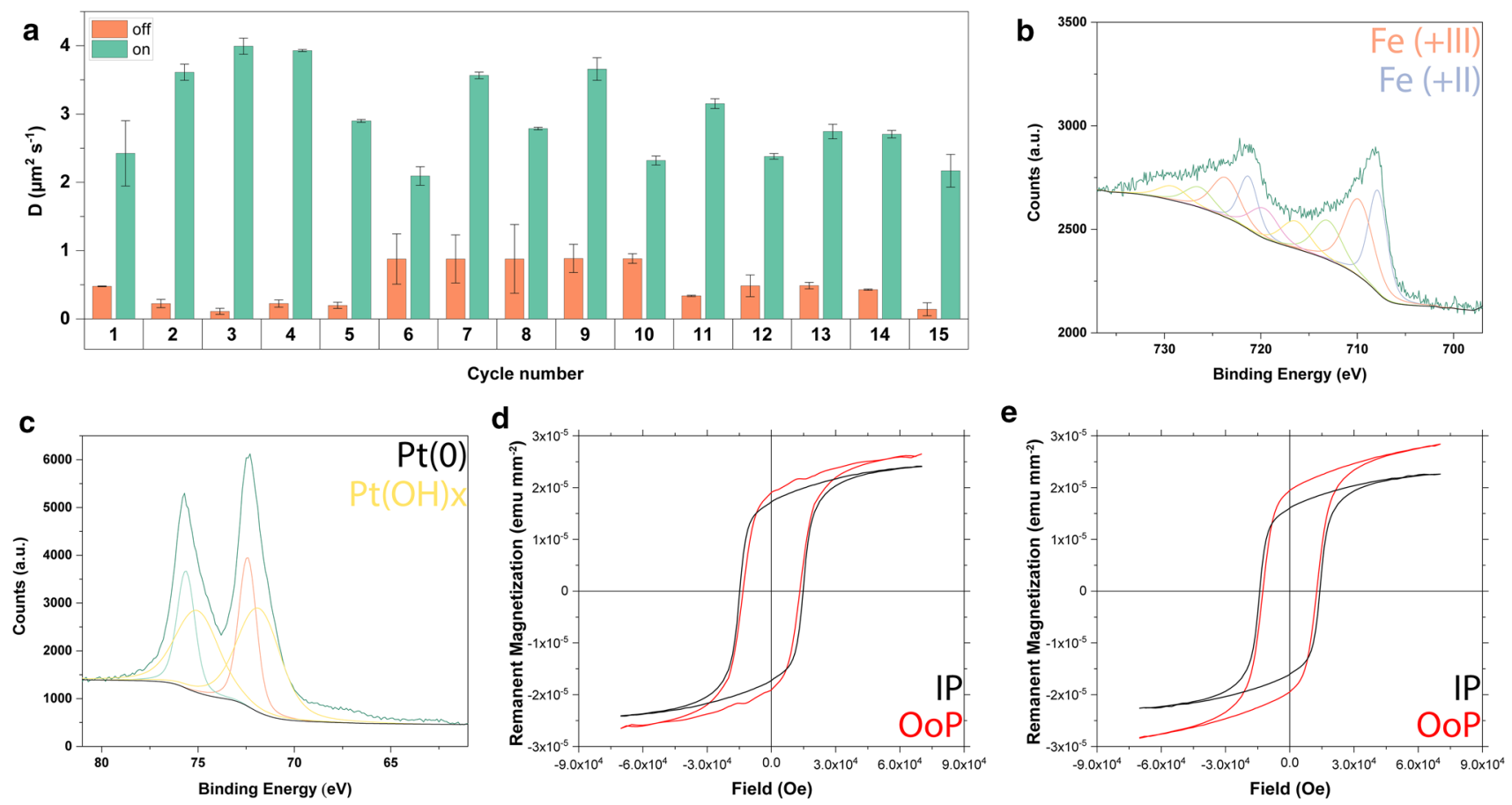

Fig. 8 a FePt-Ti-SiO 2 -based Janus particles exhibit photocatalytic behavior over the course of 15 cycles without any significant loss in switchability. b After aging in $10 \%(\mathrm{w} / \mathrm{v})$ $\mathrm{H}_{2} \mathrm{O}_{2}$, the $\mathrm{Fe}(0)$ species observed in previously disappear while $\mathrm{Fe}(+\mathrm{III})$ becomes more prevalent. c $\mathrm{Pt}(0)$ species, on the other hand become hydroxylated. The compositional changes on the surface do not, however, negatively affect FePt-Ti- $\mathrm{SiO}_{2}$-based Janus particles' magnetic properties. These stay hard magnetic $\mathbf{d}$ before and e after aging the catalytic activity increased until the wafers eventually exhibited stable activities of $0.18 \pm 0.01 \mathrm{mmol} \mathrm{cm}-2$ $\min ^{-1}$ post-aging (Supplementary Video 7). The activities for these wafers could not be switched via UV once this point was reached. We found that UV irradiation accelerates the aging process, which was also observed during the $\mathrm{O}_{2}$ evolution experiments on the Janus particles containing Ti. We thus conclude that the fabricated structures contain $\mathrm{L}_{0} \mathrm{FePt}$ in the core, but that iron oxide species and elemental platinum are also found at the surface and that these increase the catalytic activity. Their formation is accelerated by the use of UV light (e.g., a photo-accelerated catalyst).

The wafers age and over time become catalytically active irrespective whether they are illuminated with $\mathrm{UV}$ light or not. However, the FePt-Ti--SiO 2 Janus particles retain their photo-switchability over the time scale of the experiment (at least $40 \mathrm{~min}$, with $\mathrm{H}_{2} \mathrm{O}_{2}$ concentrations of up to 5\%), as shown in Fig. 8a. The aging thus does not seem to significantly affect the catalysis under these experimental conditions presumably due to the lower $\mathrm{H}_{2} \mathrm{O}_{2}$ concentrations and shorter time spans.

\subsection{Cell viability}

Another very attractive property of $\mathrm{L}_{0} \mathrm{FePt}$ is that it is not cytotoxic [12], such that FePt-based micro- and nanodevices are ideal for eventual application in vitro or in vivo biomedical settings. Since the details of the fab- rication have been changed for these new structures, we also re-examined cell viability. We could show that the FePt microswimmers of this work do not exhibit any significant cytotoxicity as shown in Fig. 9a. For this, ARPE-19 cells (positive and negative sample Fig. 9b, c respectively) were incubated with helices at a 1:100 ratio and cell viability was unaffected by the presence of both helical (Fig. 9d) and Janus particle microswimmers (Fig. 9e) after 3 days.

\section{Conclusion}

In this work, we investigated $\mathrm{L} 1_{0}$ FePt as a versatile platform for the construction of biocompatible microswimmers. As shown in earlier work, $\mathrm{L} 1_{0} \mathrm{FePt}$ is a viable material for future application in biomedical micro- and nanodevices since it is not only noncytotoxic, but also rare earth-free, while exhibiting hard magnetic properties after annealing. High coercivities in the range of $\sim 1.5 \mathrm{~T}$ make them hard to re-magnetize while their high magnetic remanence $\left(333 \mathrm{emu} \mathrm{cm}^{3}\right)$ allows for their actuation through biologically relevant fluids such as cell media via the application of relatively weak $\sim 1-4 \mathrm{mT}$ magnetic fields.

We found that coating $\mathrm{SiO}_{2}$ helices with $50 \mathrm{~nm}$ FePt thin films at lower incidence angles $\left(18^{\circ}\right)$ led to an overall increase in magnetic remanence when compared to thicker $500 \mathrm{~nm}$ films grown at glancing angles. 
Fig. 9 a Cell death as quantified by red fluorescent dead cell stain intensity after 1 and 3 days incubation for ARPE-19 cells with helical magnetic, Janus particle microswimmers, and positive (fixed cells) and negative controls $(100 \mu \mathrm{L}$ phosphate-buffered saline $(\mathrm{PBS}))$, respectively. Corresponding fluorescence microscopy images after 3 days for $\mathbf{b}$ positive, $\mathbf{c}$ negative, $\mathbf{d}$ helices and $\mathbf{e}$ Janus particle samples. Cells were stained with DAPI (blue) and LIVE DEAD cell stain (red). Scale bar is $50 \mu \mathrm{m}$
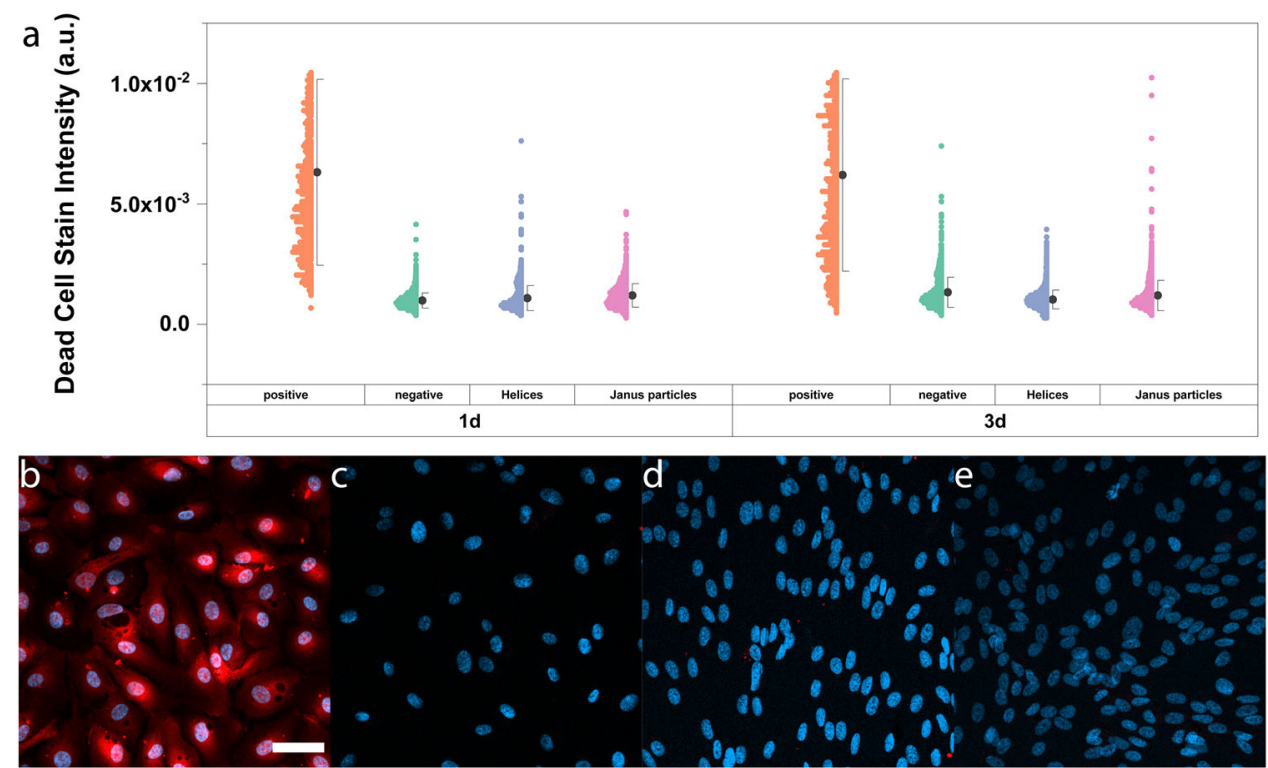

Magnetic performance and maneuverability of resulting microswimmers remained unaffected.

Depositing $\mathrm{L}_{0}$ FePt onto $1 \mu \mathrm{m} \mathrm{SiO}_{2}$ beads with a $5 \mathrm{~nm}$ Ti adhesion layer generated Janus particles, comparable to $\mathrm{Pt}-\mathrm{SiO}_{2}$-based Janus microswimmers. Asdeposited FePt catalyzed the decomposition of $\mathrm{H}_{2} \mathrm{O}_{2}$ at a slower rate than pure platinum. The catalytic activity of FePt was further reduced after annealing. However, the activity increased upon UV irradiation. UV light can thus be used to realize photo-switchable, magnetically guided $\mathrm{L}_{0}$ FePt Janus particles. We found that titanium-free $\mathrm{L}_{0}$ FePt Janus particles exhibited an irreversible aging effect which over time restored postannealing catalytic activities of up to $180 \mu \mathrm{mol} \mathrm{cm} \mathrm{cm}^{-2}$ $\min ^{-1}$. This effect has, to our knowledge, not been shown previously. Aging was photo-accelerated with UV even in the absence of titanium. Certainly, the use of hydrogen peroxide as a fuel is problematic for eventual application, replacing this fuel could therefore be subject of further investigations. However, chemically propelled microswimmers offer the possibility of realizing chemotaxis [36].

Supplementary information The online version contains supplementary material available at https://doi.org/ 10.1140/epje/s10189-021-00074-1.

Acknowledgements P.F. and M.B. acknowledge funding from the DFG (Projekt No. 253407113 under the SPP program 1726). HNB would like to thank the Minerva Stiftung for funding. SNK thanks the International Max Planck Research School for Intelligent Systems (IMPRSIS) for support. The authors thank Cornelia Miksch, Helmut Kammerlander, and Kersten Hahn and Peter van Aken for Langmuir-Blodgett fabrication, quartz ampoule fabrication, and EDX measurements, respectively. We thank Dr. Sven Schnichel's laboratory at the Tübingen University Hospital for providing the ARPE-19 cell line and Prof. Dr. Joachim Spatz for access to scanning electron microscopes.
The authors would like to thank an anonymous reviewer for suggesting control experiments without Ti.

Funding Open Access funding enabled and organized by Projekt DEAL.

\section{Author contribution statement}

VMK, JPG, HNB and PF conceptualized this work. VMK and HNB fabricated the microswimmers. VMK performed the cell culture experiments. JPG performed the $\mathrm{O}_{2}$ evolution experiment and scanning electron microscopy. FP performed the particle tracking microscopy of the Janus microswimmers and the transmission electron microscopy. SNK analyzed the particle tracking microscopy data. VMK and RG performed the particle tracking and guidance microscopy of the magnetic microswimmers. KS performed the SQUID experiments. HNB performed the XPS experiments. MB, MAC and PF supervised this work. All authors contributed to the final version of the manuscript.

Data Availability Statement This manuscript has data included as electronic supplementary material. [Authors' comment: The online version of this article contains supplementary material, which is available to authorized users.]

Open Access This article is licensed under a Creative Commons Attribution 4.0 International License, which permits use, sharing, adaptation, distribution and reproduction in any medium or format, as long as you give appropriate credit to the original author(s) and the source, provide a link to the Creative Commons licence, and indicate if changes were made. The images or other third party material in this article are included in the article's Creative Commons licence, unless indicated otherwise in a credit line to the material. If material is not included in the article's Creative Commons 
licence and your intended use is not permitted by statutory regulation or exceeds the permitted use, you will need to obtain permission directly from the copyright holder. To view a copy of this licence, visit http://creativecomm ons.org/licenses/by/4.0/.

\section{References}

1. D. Bobo, K.J. Robinson, J. Islam, K.J. Thurecht, S.R. Corrie, Pharm. Res. 33(10), 2373 (2016)

2. M.J. Rohovie, M. Nagasawa, J.R. Swartz, Bioeng. Transl. Med. 2(1), 43 (2017)

3. E.M. Purcell, Am. J. Phys. 45(1), 3 (1977)

4. T. Qiu, T.-C. Lee, A.G. Mark, K.I. Morozov, R. Münster, O. Mierka, S. Turek, A.M. Leshansky, P. Fischer, Nat. Commun. 5(1), 1 (2014)

5. S. Palagi, A.G. Mark, S.Y. Reigh, K. Melde, T. Qiu, H. Zeng, C. Parmeggiani, D. Martella, A. Sanchez-Castillo, N. Kapernaum, Nat. Mater. 15(6), 647 (2016)

6. A. Ghosh, P. Fischer, Nano Lett. 9(6), 2243 (2009)

7. L. Zhang, J.J. Abbott, L. Dong, B.E. Kratochvil, D. Bell, B.J. Nelson, Appl. Phys. Lett. 94(6), 064107 (2009)

8. D. Schamel, A.G. Mark, J.G. Gibbs, C. Miksch, K.I. Morozov, A.M. Leshansky, P. Fischer, ACS Nano 8(9), 8794 (2014)

9. D. Walker, B.T. Käsdorf, H.-H. Jeong, O. Lieleg, P. Fischer, Sci. Adv. 1(11), e1500501 (2015)

10. Z. Wu, J. Troll, H.-H. Jeong, Q. Wei, M. Stang, F. Ziemssen, Z. Wang, M. Dong, S. Schnichels, T. Qiu, P. Fischer, Sci. Adv. 4(11), eaat4388 (2018)

11. M. Ermolli, C. Menné, G. Pozzi, M.-Á. Serra, L.A. Clerici, Toxicology 159(1-2), 23 (2001)

12. V.M. Kadiri, C. Bussi, A.W. Holle, K. Son, H. Kwon, G. Schütz, M.G. Gutierrez, P. Fischer, Adv. Mater. 32(25), 2001114 (2020)

13. Y. Shi, M. Lin, X. Jiang, S. Liang, J. Nanomater. 2015 (2015)

14. K. Son, G. Ryu, H.-H. Jeong, L. Fink, M. Merz, P. Nagel, S. Schuppler, G. Richter, E. Goering, G. Schütz, Small 15(34), 1902353 (2019)

15. J.L. Moran, J.D. Posner, Annu. Rev. Fluid Mech. 49(1), $511(2017)$

16. D. Walker, Magnetically Actuated Micro-and Nanopropellers (Cuvillier Verlag, 2015)
17. M.M. Hawkeye, M.T. Taschuk, M.J. Brett, Glancing Angle Deposition of Thin Films: Engineering the Nanoscale (Wiley, 2014)

18. U. Choudhury, L. Soler, J.G. Gibbs, S. Sanchez, P. Fischer, Chem. Commun. 51(41), 8660 (2015)

19. H. Yu, A. Kopach, V.R. Misko, A.A. Vasylenko, D. Makarov, F. Marchesoni, F. Nori, L. Baraban, G. Cuniberti, Small 12(42), 5882 (2016)

20. D. Allan, C. van der Wel, N. Keim, T.A. Caswell, D. Wieker, R. Verweij, C. Reid, L. Grueter, K. Ramos, R.W. Perry, et al. Soft-matter trackpy: Trackpy v0. 4.2 (2019). https://doi.org/10.5281/zenodo.3492186

21. J.C. Crocker, D.G. Grier, J. Colloid Interface Sci. $\mathbf{1 7 9}(1), 298$ (1996)

22. J.R. Howse, R.A.L. Jones, A.J. Ryan, T. Gough, R. Vafabakhsh, R. Golestanian, Phys. Rev. Lett. 99(4), 048102 (2007)

23. A.E. Carpenter, T.R. Jones, M.R. Lamprecht, C. Clarke, I.H. Kang, O. Friman, D.A. Guertin, J.H. Chang, R.A. Lindquist, J. Moffat, Genome Biol. 7(10), 1 (2006)

24. R. Streubel, P. Fischer, F. Kronast, V.P. Kravchuk, D.D. Sheka, Y. Gaididei, O.G. Schmidt, D. Makarov, J. Phys. D Appl. Phys. 49(36), 363001 (2016)

25. N. Kikuchi, S. Okamoto, O. Kitakami, J. Appl. Phys. 103(7), 07D511 (2008)

26. Y. Man, E. Lauga, Phys. Fluids 25(7), 071904 (2013)

27. S. Hsieh, P.-Y. Lin, J. Nanopart. Res. 14(6), 956 (2012)

28. C. Richard, J. Simmchen, A. Eychmüller, Z. Phys. Chem. 232(5-6), 747 (2018)

29. M. Malakootian, M.R. Heidari, Z. Phys. Chem. 1 (ahead-of-print) (2020)

30. J. Giltinan, V. Sridhar, U. Bozuyuk, D. Sheehan, M. Sitti, Adv. Intell. Syst. 3(1), 2000204 (2021)

31. Y.-T. Liu, Q.-B. Yuan, D.-H. Duan, Z.-L. Zhang, X.-G. Hao, G.-Q. Wei, S.-B. Liu, J. Power Sources 243, 622 (2013)

32. J. Palacci, S. Sacanna, A.P. Steinberg, D.J. Pine, P.M. Chaikin, Science 339(6122), 936 (2013)

33. D.P. Singh, W.E. Uspal, M.N. Popescu, L.G. Wilson, P. Fischer, Adv. Funct. Mater. 28(25), 1706660 (2018)

34. V. Sridhar, B.-W. Park, M. Sitti, Adv. Funct. Mater. 28(25), 1704902 (2018)

35. D. Zhou, L. Ren, Y.C. Li, P. Xu, Y. Gao, G. Zhang, W. Wang, T.E. Mallouk, L. Li, Chem. Commun. 53(83), 11465 (2017)

36. M.N. Popescu, W.E. Uspal, C. Bechinger, P. Fischer, Nano Lett. 18(9), 5345 (2018) 